\title{
Massive Star Formation: New Results
}

\author{
Ed Churchwell \\ Washburn Observatory, University of Wisconsin, 475 North Charter \\ Street, Madison, WI 53706
}

\section{Introduction}

In the past year several new observations with important implications for massive star formation (MSF) have been obtained. Among these were the detection of UC HII region precursor candidates at $350 \mu \mathrm{m}$ and the discovery of many hard $\mathrm{X}$-ray point sources in the Orion and W3 MSF regions. These observations are summarized below.

\section{Precursors of UC HII Regions}

Hunter et al. $(1998,2000)$ imaged $25 \mathrm{MSF}$ regions at $350 \mu \mathrm{m}$ to search for candidate precursors of UC HII regions (i.e. luminous submm/FIR emission, maser sources, and no HII emission). Of the 28 sources detected, 10 appear to be UC HII precursor candidates.

Figure 1 shows an example of one (IRAS18317-0757 SMM2).

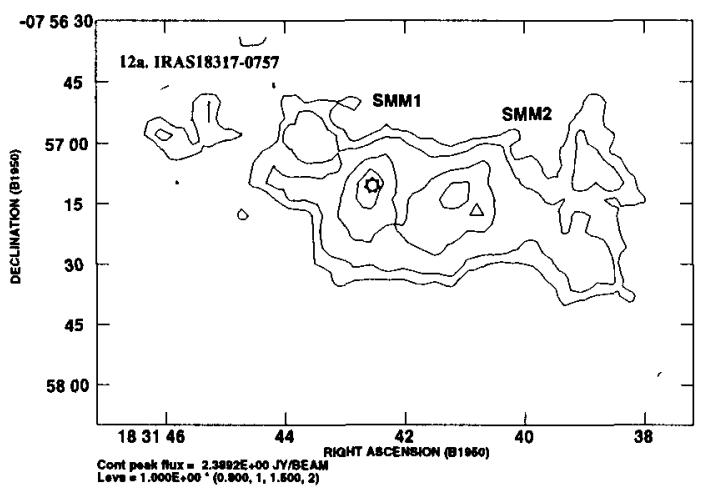

Figure 1.

IRAS18317-0757 SMM1 is centered on the UC HII region G23.96+0.15, SMM2 has an $\mathrm{H}_{2} \mathrm{O}$ maser and no $\mathrm{HII}$ region. The mean physical properties (derived from grey body models) of the $28350 \mu \mathrm{m}$ "hot cores" found by Hunter et al. (2000) are given in Table 1.

Table 1. Mean physical properties of "hot cores" (Hunter et al. 2000)

\begin{tabular}{rrr} 
& Range & Mean \\
\hline $\log \mathrm{L} / L_{\odot}$ & $4.3-6.1$ & 5.0 \\
$\log \mathrm{M} / M_{\odot}$ & $2.4-5.0$ & 3.5 \\
$\log \mathrm{N}_{H}$ & $22.2-24.9$ & 23.8 \\
$\log (\mathrm{L} / \mathrm{M})$ & $0.9-2.7$ & 1.5
\end{tabular}

The main conclusions are that MSF requires core masses $>10^{3} M_{\odot}$ and densities $\geq 10^{5} \mathrm{~cm}^{-3}$. 


\section{X-Ray Emission from MSF Regions}

Hofner, Churchwell, and Whitney (2000), using the high spatial resolution and sensitivity of Chandra, found that the "extended, hard X-ray emission" observed previously with ASCA by Hofner and Churchwell (1997), resolves into several hundred X-ray point sources distributed over the entire W3 complex. The sources at the core of W3 are very energetic with most of their energy emitted in the 3-7.5 KeV range. Their hardness is enhanced by extinction of softer $\mathrm{X}$-rays by over-lying hydrogen.

$\mathrm{X}$-ray sources lie at the peak of most of the UC HII regions in W3. The locations of the X-ray sources suggests that they may be the ionizing star of the UC HII components, although this still remains to be established. There are also many X-ray sources that do not coincide with radio sources. The nature of these sources are not yet known. They could be T Tau stars (i.e. low-mass pre-main sequence stars), interacting close binaries, stars with strong coronal activity, etc. Unfortunately, not enough data on these objects exist to establish their nature.

The NIR sources of Megeath et al. (1996) and the X-ray sources in the core of W3 show very few coincidences. The NIR sources are uniformly distributed in the observed field with little increase in density toward the UC HII regions. The NIR sources appear to be objects located on the near side of the W3 molecular cloud and may have little to do with the deeply embedded UC HII regions. This is not the case for the hard X-ray sources. This leads one to the interesting speculation that perhaps the best way to locate the ionizing stars of UC HII regions is in the 2-8 Kev X-ray band, not in the NIR.

In summary, MSF regions are rich in hard X-ray sources, which probably represent a variety of stellar types. The W3 study is also consistent with the earlier detection of many X-ray sources in the Orion MSF region by Yamauchi and Koyama (1993) and spectacularly confirmed by Garmire et al. (2000) using Chandra.

\section{References}

Garmire, G., Feigelson, E. D., Broos, P., Hillenbrand, L. A., Pravdo, S. H., Townsley, L., Tsuboi, Y. 2000, AJ, in press.

Hofner, P., Churchwell, E. 1997, Ap. J. Lett., 486, L39

Hofner, P., Churchwell, E., Whitney, B. 2000, in preparation

Hunter, T. R., Churchwell, E., Watson, C., Cox, P., Benford, D.J., Roelfsema, P.R. 2000, AJ, 119, 2711

Hunter, T. R., Neugebauer, G., Benford, D. J., Matthews, K., Lis, D. C., Serabyn, E., Phillips, T. G. 1998, ApJ, 493, 97

Megeath, S. T., Herter, T., Beichman, C., Gautier, N., Hester, J. J., Rayner, J., Shupe, D. 1996, A\& A, 307, 775

Yamauchi, S., Koyama, K. 1993, ApJ, 405, 268 